\title{
Flat lensing in the visible frequency range by woodpile photonic crystals
}

\author{
L. Maigyte,${ }^{1, *}$ V. Purlys, ${ }^{2}$ J. Trull, ${ }^{1}$ M. Peckus,,${ }^{2,3}$ C. Cojocaru, ${ }^{1}$ D. Gailevičius, ${ }^{2}$ M. Malinauskas, ${ }^{2}$ and K. Staliunas ${ }^{1,4}$ \\ ${ }^{1}$ Departament de Física i Enginyeria Nuclear, Universitat Politècnica de Catalunya, Rambla Sant Nebridi 22, Terrassa 08222, Spain \\ ${ }^{2}$ Laser Research Center, Department of Quantum Electronics, Vilnius University, Saulètekio Al. 10, Vilnius LT-10223, Lithuania \\ ${ }^{3}$ Center for Physical Sciences and Technology, Savanoriu Ave. 231, Vilnius LT-02300, Lithuania \\ ${ }^{4}$ Institució Catalana de Recerca i Estudis Avançats (ICREA), Pg. Lluís Companys, 23, Barcelona 08010, Spain \\ *Corresponding author: lina.maigyte@upc.edu
}

Received March 14, 2013; revised June 3, 2013; accepted June 3, 2013; posted June 4, 2013 (Doc. ID 187073); published July 3, 2013

\begin{abstract}
We experimentally demonstrate full two-dimensional focalization of light beams at visible frequencies by a three-dimensional woodpile photonic crystal. The focalization (the flat lensing) with focal distances of the order of 50-70 $\mu \mathrm{m}$ is experimentally demonstrated. Experimental results are compared with numerical calculations and interpreted by harmonic expansion studies. (c) 2013 Optical Society of America

OCIS codes: (050.5298) Photonic crystals; (230.5298) Photonic crystals.

http://dx.doi.org/10.1364/OL.38.002376
\end{abstract}

Spatial periodic modulation of the refractive index on a wavelength scale in photonic crystals (PhCs), provides control of both temporal and spatial dispersion properties of light waves, in the latter case offering the possibility of manipulating the spatial propagation of the light beams. PhCs may provide, for particular geometries, anomalous spatial dispersion for a beam propagating inside the structure [1] , leading to nondiffractive (selfcollimated) propagation of light [2] and negative refrac-

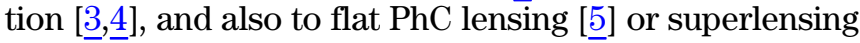
[6] effects.

The concept of flat PhC lensing is based on the transformation of the phases of the angular field components. The convex-curved phase shifts of field components accumulated during propagation inside the $\mathrm{PhC}$ can be compensated by the usual concave-curved phase shifts during propagation in a homogeneous material, both in front of and behind the $\mathrm{PhC}$, resulting in focusing behind the PhC. The distance between the object and the $\mathrm{PhC}, l_{1}$, and between the $\mathrm{PhC}$ and the image, $l_{2}$, [Fig. 1(a)] obey the relation $l_{1}+l_{2}=f$, where $f$ is the focal distance of the flat $\mathrm{PhC}$ lens. This is in contrast to the usual focusing by conventional or by Fresnel lenses, where the well-known relation $1 / l_{1}+1 / l_{2}=1 / f$ holds.

In this Letter we experimentally demonstrate full two-dimensional (2D) focusing by a polymer-based threedimensional (3D) woodpile PhC. Full 2D flat lens focusing has been experimentally shown for microwaves [7] and for sound waves []. Moreover, even 1D focusing/imaging by PhC slabs has thus far been experimentally demonstrated only in the near-IR frequency range [9].

$\mathrm{PhC}$ lensing is usually considered for modulation periods of the order of wavelength. Flat lensing occurs due to the convex-curved spatial dispersion (or isofrequency) lines in the first, or at most in the second, propagation band. In particular, for PhCs of square symmetry, the corner of the Brillouin zone (BZ) is positioned at $\lambda=d_{0} \bar{n}(\lambda$ is the wavelength, $\bar{n}$ is the effective refractive index of the $\mathrm{PhC}$, and $d_{0}$ is the lattice period). The self-collimation (SC) (the flattening of the spatial dispersion lines) occurs at frequencies below the corner of the BZ, i.e., at $\lambda>d_{0} \bar{n}$. The flat lensing, which is based on anomalously curved spatial dispersion lines, generally occurs between the frequencies of SC and of the edge of the BZ. The experimental demonstration of flat lensing in the visible range is therefore a difficult task, due to technological limitations of PhC fabrication techniques at this scale. We use an alternative approach based on PhCs with relatively large modulation periods, but searching the flat lensing effects in higher order bands. This, on one hand, simplifies the fabrication of the samples, but on the other hand makes the observation and interpretation of the
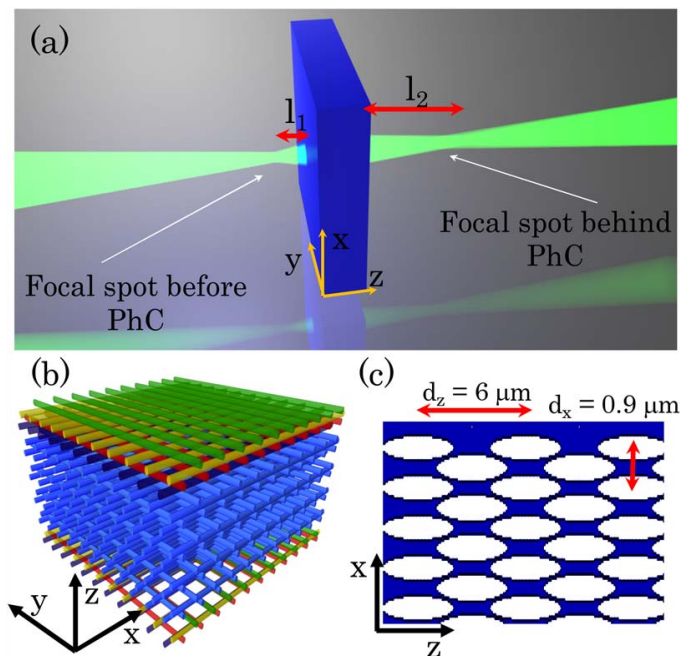

(c)
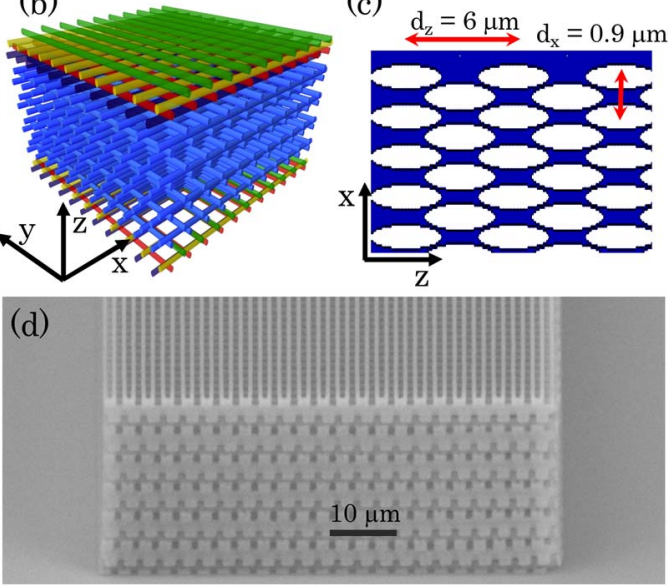

Fig. 1. (a) Flat $\mathrm{PhC}$ lensing scheme, (b) schematic representation of a woodpile $\mathrm{PhC}$, (c) reduced 2D structure, and (d) a scanning electron microscope image of the $\mathrm{PhC}-45^{\circ}$ oblique view. 
focalization effect more complicated, since the harmonic expansion techniques become very complicated in highorder propagation bands.

Finite-difference time-domain (FDTD) calculations were first performed in order to check the expected focusing of a Gaussian beam. As the woodpile structure [Fig. 1(b)] consists of bars directed along the $x$ and $y$ directions in alternating order, the $3 \mathrm{D}$ refractive index profile can be expressed as a sum of $2 \mathrm{D}$ profiles: $\Delta n(x, y, z)=\Delta n_{x}(x, z)+\Delta n_{y}(y, z)$. This particular symmetry allows the factorization of the 3D wave envelopes, $A(x, y, z)=A_{x}(x, z) \cdot A_{y}(y, z)$, where both field quadratures propagate independently of each other. This factorization (see [10] for details) allows us to apply the $2 \mathrm{D}$ calculations for simulation of the beam propagation through full 3D crystal.

The reduced 2D index profile [see Fig. 1(c)] consists of elliptically shaped rods of refractive index $n=1.5$ embedded in air. The parameters were as follows: transverse period $d_{x}=0.9 \mu \mathrm{m}$, longitudinal period $d_{z}=6 \mu \mathrm{m}$ (filling factor 70\%), transverse size $80 \mu \mathrm{m}$, and length of the crystal $L \sim 30 \mu \mathrm{m}$ (5 longitudinal periods). The corner of the $\mathrm{BZ}$ is estimated at $\lambda_{\mathrm{BZ}}=370 \mathrm{~nm}$. The beam was focused just at the front face of the $\mathrm{PhC}$, with the focal spot width in the range of 1.6-3.0 $\mu \mathrm{m}$.

The periodic modulation of the refraction index couples harmonic components of the field and locks them mutually to the Bloch modes. Following [10] we assume that the interaction can be described by the three most relevant field harmonics: the central component with the carrier wave vector $\vec{k}_{0}$ and the modulated components with wave vectors $\vec{k}_{0}+\vec{q}_{1,2}$, where $\vec{q}_{1,2}=\left( \pm q_{x},-q_{z}\right)$ are the relevant (near-resonant) wave vectors of the refractive index modulation.

The resonant interaction between the harmonic field components spanned by $\vec{q}_{1,2}=\left( \pm q_{x},-q_{z}\right)$ is given by the condition $2 q_{z} k_{0} \bar{n} / q_{x}^{2} \equiv 2 d_{x}^{2} \bar{n} /\left(d_{z} \lambda\right)=1$ in paraxial treatment. This defines the wavelength corresponding to the edge of $\mathrm{BZ}: \lambda_{\mathrm{BZ}}=2 d_{x}^{2} \bar{n} / d_{z}$. The $\mathrm{SC}$ regime is obtained for the frequencies below the resonance, $\lambda_{\mathrm{SC}}>\lambda_{\mathrm{BZ}}$, depending on the amplitude of index modulation. For frequencies between those of SC and the edge of the BZ, $\lambda_{\mathrm{BZ}}<\lambda<\lambda_{\mathrm{SC}}$, the spatial dispersion curves become convex, and flat lens focusing can be expected.

First we identified the SC wavelength, by propagating the narrow beam inside the elongated woodpile structure (20 longitudinal periods), at around $\lambda_{\mathrm{SC}}=610 \mathrm{~nm}$. We concentrated, consequently, on the range $370 \mathrm{~nm}<\lambda<$ $610 \mathrm{~nm}$, according to the previous analysis. The results are summarized in Fig. 2. The beam of $532 \mathrm{~nm}$ wavelength focuses at a distance of around $40 \mu \mathrm{m}$, while the $570 \mathrm{~nm}$ beam has the smallest width at a distance of around $70 \mu \mathrm{m}$ behind the PhC. For the wavelength of $780 \mathrm{~nm}$ [Fig. 2(e)], which is already in the normal spatial dispersion regime according to the estimation above, no focusing was obtained.

Woodpile PhC samples, with the parameters used in the above FDTD calculations, were fabricated by laser direct writing technique [11]. A hybrid organic-inorganic sol-gel photopolymer prepared as in [12] was used, except the molar ratio of 3-methacryloxypropyltrimethoxysilane to Zirconium (IV) isopropoxide was chosen
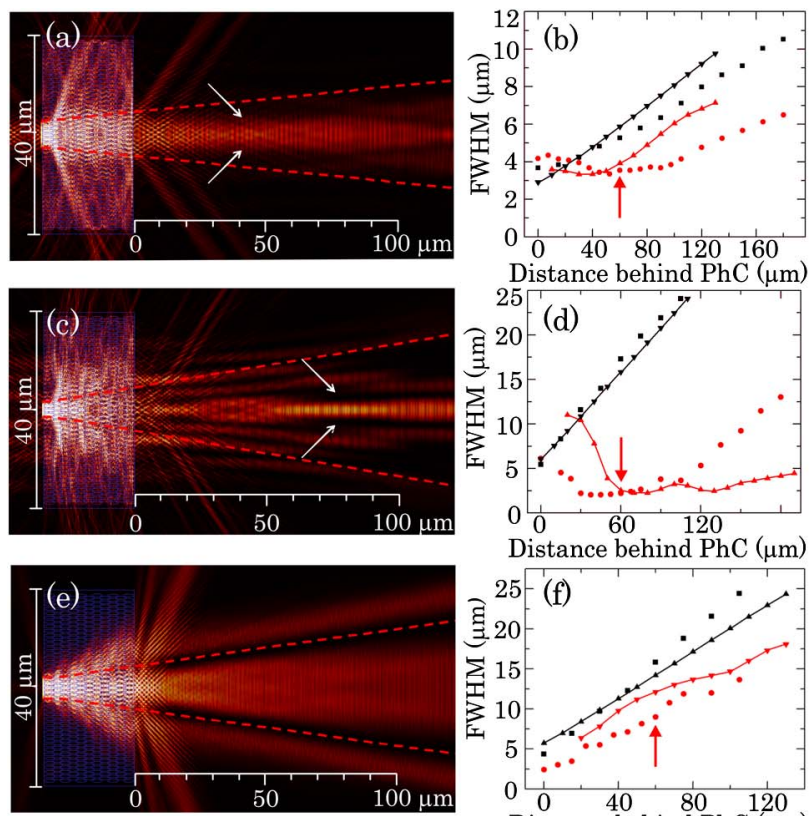

Distance behind $\mathrm{PhC}(\mu \mathrm{m})$
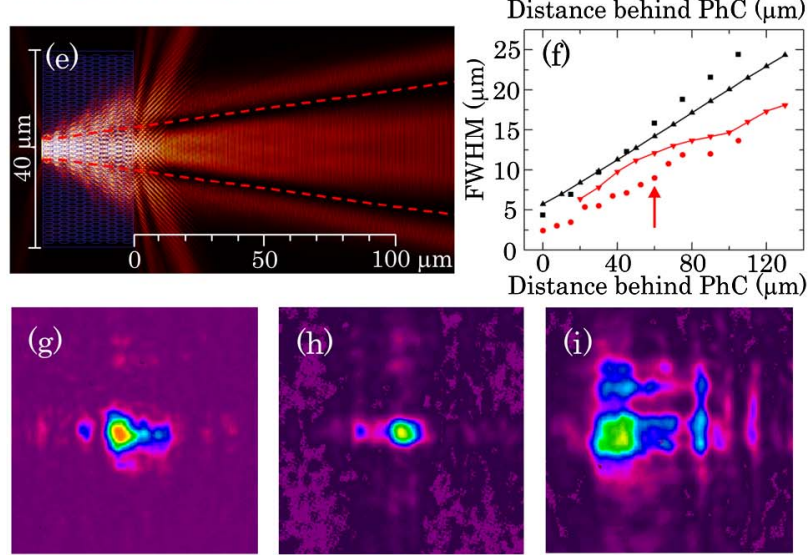

Fig. 2. (a), (c), (e) Field intensity distributions at 532, 570, and $780 \mathrm{~nm}$, inside and behind the PhC, calculated by 2D FDTD; (b), (d), (f) the beam width depending on distance behind the crystal (red) compared to the width of the reference beam (black) (dots, experimental results; lines, numerical results); (g)-(i) $2 \mathrm{D}$ distributions of the beams at the plane of $60 \mu \mathrm{m}$ behind the PhC taken with a CCD camera for the corresponding wavelengths of 532, 570, and $700 \mathrm{~nm}$ [corresponding positions are marked by arrows in (b), (d), and (f), respectively].

as 5:3, and thioxanthen-9-one was used as the photoinitiator. PhCs were written employing point-by-point irradiation of the sample by femtosecond laser pulses (300 fs pulse duration, $515 \mathrm{~nm}$ wavelength, $200 \mathrm{kHz}$ repetition rate). A sample translation speed of $500 \mu \mathrm{m} / \mathrm{s}$ and laser power of $40 \mu \mathrm{W}$ (after the objective) were chosen. The beam was tightly focused by a $63 \times 1.4 \mathrm{NA}$ lens. An example of the resulting $\mathrm{PhC}$ structure is shown in Fig. 1(d).

For the experimental measurements a supercontinuum source pumped by an $800 \mathrm{~nm}$ Ti:saphire laser was used. The particular wavelengths of 532, 570 , and $700 \mathrm{~nm}$ were selected by a monochromator. The beams were focused using a $20 \times 0.4 \mathrm{NA}$ microscope objective onto the front face of the $\mathrm{PhC}$, which for different frequencies resulted in waist widths varying from 1.6 to $3 \mu \mathrm{m}$. The beam profiles at different distances behind the crystal were recorded with a CCD camera mounted, together with the imaging system, on a translational stage. The width of the beam behind the $\mathrm{PhC}$ was calculated, applying a Gaussian fit to the CCD images. The experimental results are summarized and compared with the FDTD calculation results in Fig. 2. Quantitative correspondence between the experimental and numerical data is not perfect, but the qualitative tendencies are the same. 


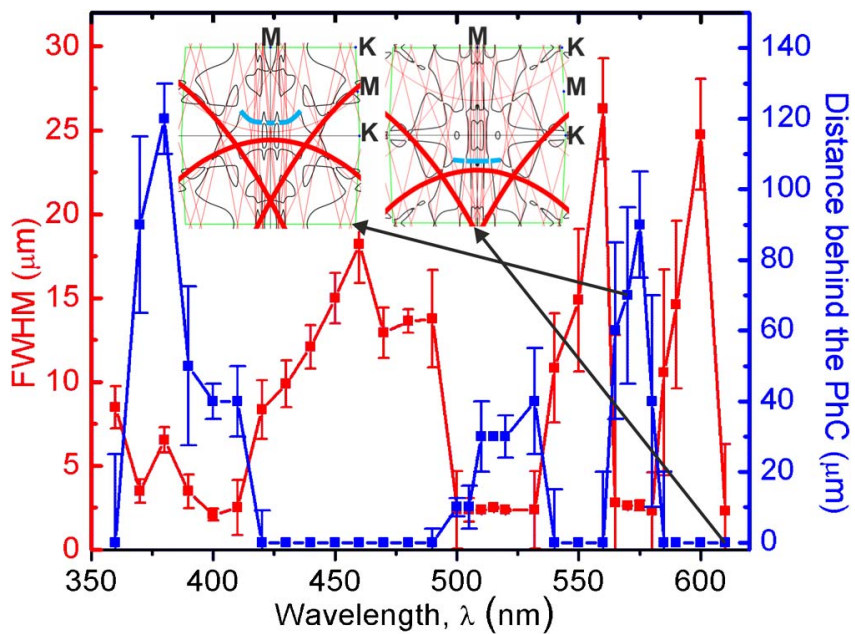

Fig. 3. Minimum diameter of the beam behind the PhC (red) and the focal distance (blue) versus wavelength calculated by FDTD. The two insets represent isofrequency lines, where the spanning harmonics (see main text) are highlighted in red and global trends of isofrequency are lines highlighted in blue. The left inset is at the frequency of flat lensing (at $570 \mathrm{~nm}$ ) and the right one at self-collimation (at $610 \mathrm{~nm}$ ).

The smallest beam diameter is found at particular distances (around $50 \mu \mathrm{m}$ ) behind the $\mathrm{PhC}$ at 532 and $570 \mathrm{~nm}$, while monotonous spreading is observed at $700 \mathrm{~nm}$. However, even in the latter case the divergence was slightly less than that of the reference beam, due to the spatial filtering of the beam. The minimum widths of the beam behind the $\mathrm{PhC}$ were around 2.1 and $3.4 \mu \mathrm{m}$ at 570 and $532 \mathrm{~nm}$, respectively. This result gives a rough estimation of the numerical aperture of the flat lens: $\mathrm{NA} \approx 0.1$.

We note that we could not observe the beam focalization continuously throughout the expected wavelength range $\lambda_{\mathrm{BZ}}<\lambda<\lambda_{\mathrm{SC}}$, but just in several distinct areas of this range. Figure 3 shows the focalization performance calculated by FDTD (the beam diameter at the focus as well the focal length depending on the wavelength). The reason for that is that we worked at very high-order propagation bands; although the character of the isofrequency lines is globally defined by the first harmonic of the index modulation, the higher harmonics interfere and distort the isoline picture. The higher the propagation band is, the more harmonics come into play, resulting in a stronger distortion of the isoline picture. As seen from the insets in Fig. 3, although the isoline picture (calculated by the standard plane wave expansion method) is quite disordered, the global trends can still be traced (highlighted in blue), indicating the appearance of flat segments (for SC) as well as positively curved segments (for flat lensing). The spanning harmonics are highlighted in red.

To conclude, we have reported the first (to our knowledge) experimental confirmation of beam focusing by a flat $3 \mathrm{D}$ woodpile $\mathrm{PhC}$ at visible frequencies. The results show a convincing flat lensing with focal distances of around $50-70 \mu \mathrm{m}$ behind the crystal. No focusing appears at other wavelengths, excluding all possible geometrical focusing effects (e.g., due to possibly curved surfaces of the woodpile). The observations are in good correspondence with the 2D FDTD simulations.

The work was supported by the Spanish Ministerio de Ciencia e Innovación and European Union FEDER (project FIS2011-29734-C02-01), the European Union Structural Funds project "Postdoctoral Fellowship Implementation in Lithuania," and the Research Council of Lithuania project grant “Mikrošviesa” (No. MIP-12241).

\section{References}

1. C. Luo, S. G. Johnson, J. D. Joannopoulos, and J. B. Pendry, Phys. Rev. B 65, 201104 (2002).

2. H. Kosaka, T. Kawashima, A. Tomita, M. Notomi, T. Tamamura, T. Sato, and S. Kawakami, Appl. Phys. Lett. 74, 1212 (1999).

3. E. Cubukcu, K. Aydin, E. Ozbay, S. Foteinopoulou, and C. M. Soukoulis, Nature 423, 604 (2003).

4. J. Serbin and M. Gu, Opt. Express 14, 3563 (2006).

5. P. V. Parimi, W. T. Lu, P. Vodo, and S. Sridhar, Nature 426, 404 (2003).

6. E. Cubukcu, K. Aydin, E. Ozbay, S. Foteinopoulou, and C. M. Soukoulis, Phys. Rev. Lett. 91, 207401 (2003).

7. Z. Lu, J. A. Murakowski, C. A. Shuetz, S. Shi, G. J. Schneider, and D. W. Prather, Phys. Rev. Lett. 95, 153901 (2005).

8. A. Cebrecos, V. Romero-Garcia, R. Pico, I. Perez-Arjona, V. Espinosa, V. J. Sanchez-Morcillo, and K. Staliunas, J. Appl. Phys. 111, 104910 (2012).

9. E. Schonburn, T. Yamashita, W. Park, and C. J. Summers, Phys. Rev. B 73, 195117 (2006).

10. K. Staliunas and R. Herrero, Phys. Rev. E 73, 016601 (2006).

11. M. Deubel, G. Freymann, M. Wegener, S. Pereira, K. Bush, and C. M. Soukoulis, Nat. Mater. 3, 444 (2004).

12. A. Ovsianikov, J. Viertl, B. Chickov, M. Oubaha, B. MacCraith, I. Sakellari, A. Guakoumaki, D. Gray, M. Vamvakaki, M. Farsari, and C. Fotakis, ACS Nano 2, 2257 (2008). 